\title{
The Performance of an in-house nested-PCR technique for pleural tuberculosis diagnoses
}

\author{
Lílian Maria Lapa Montenegro ${ }^{[1]}$, Bruno César da Silva ${ }^{[2]}$, Juliana Figueirêdo da Costa Lima ${ }^{[1]}$, \\ Heidi Lacerda Alves da Cruz ${ }^{[1]}$, Rosana de Albuquerque Montenegro ${ }^{[1]}$, \\ Fernando Luiz Cavalcanti Lundgren ${ }^{[3]}$, Alfredo Pereira Leite de Albuquerque Filho ${ }^{[2]}$ \\ and Haiana Charifker Schindler ${ }^{[1]}$
}

[1]. Departamento de Imunologia, Centro de Pesquisas Aggeu Magalhães, Recife, PE. [2]. Departamento de Clínica Médica, Hospital Barão de Lucena, Recife, PE. [3]. Hospital Geral Otávio de Freitas, Recife, PE.

\begin{abstract}
Introduction: This study evaluated the performance of an in-house nested-PCR system for the detection of the Mycobacterium tuberculosis complex in pleural fluid, blood and urine samples from pleural effusion tuberculosis patients by health services physicians in Pernambuco, Brazil. Methods: A prospective double-blind study with 37 hospitalized patients of both sexes, aged over 15, was used to investigate the diagnosis of pleural effusion. The criteria used to define the cases included the demonstration of bacillus in biological samples by smear or culture or by a granulomatous finding in the histopathological examination, associated with an evident response to specific treatments to each clinical situation. Pleural fluid, blood and urine samples were collected and subjected to routine tests and the nested PCR technique to assess for $M$. tuberculosis amplification. Results: In total, 37 pleural effusion patients took part in the study, of whom $19(51.3 \%)$ had tubercular etiologies and 18 (48.7\%) had etiologies from other causes. When the pleural fluid, blood and/or urine sample in-house nested-PCR sensitivities were evaluated simultaneously, the results were positive regardless of the biological specimen (the sensitivity was $84.2 \%$ ); however, when the blood and/or urine samples were analyzed together, the sensitivity was $72.2 \%$. When the pleural fluid samples were evaluated alone, the sensitivity was only $33.3 \%$. Conclusions: The performance of the diagnostic pleural tuberculosis nested-PCR was directly related to the diversity of the samples collected from the same patient. Additionally, this study may identify a need to prioritize non-invasive blood and urine collection for this diagnosis.
\end{abstract}

Keywords: Pleural tuberculosis. Extrapulmonary tuberculosis. Nested PCR diagnosis. Mycobacterium tuberculosis.

\section{INTRODUCTION}

According to World Health Organization (WHO) data from 2011, Brazil ranks $19^{\text {th }}$ for the highest amount of worldwide tuberculosis (TB) cases. Furthermore, 22 nations account for $80 \%$ of all worldwide TB cases; of these nations, Brazil is the only Latin American country on the list $^{1}$. In 2010, 10,170 new extrapulmonary TB cases were recorded in Brazil, which corresponded to $14 \%$ of the TB cases in the country. Additionally, 531 of these new cases occurred in the State of Pernambuco ${ }^{2}$.

However, these figures are much lower than the WHO estimates and are thus evidence of diagnostic deficiencies, under-notifications and the inefficiency of disease control programs, especially regarding the extrapulmonary form of $\mathrm{TB}^{3}$. Additionally, while the extrapulmonary TB distribution in the country is uneven, most reported cases are mainly concentrated in the Southeast and Northeast ${ }^{4}$.

Address to: Dra. Lilian Maria Lapa Montenegro. Depto Imunologia/CPqAM/ FIOCRUZ. Av. Prof. Moraes Rego s/nº, 50.670-420 Recife, PE, Brasil.

Phone: 5581 2101-2681; Fax: 5581 2101-2640

e-mail: lilian@cpqam.fiocruz.br

Received 14 June 2013

Accepted 4 October 2013
Pleural TB is the main manifestation of the extrapulmonary disease in adults and is also a frequent cause of pleural effusion (PE), which occurs in up to $30 \%$ of TB patients ${ }^{5,6}$. However, these frequencies vary in different countries in accordance with disease prevalence and positive human immunodeficiency virus (HIV) serologies ${ }^{7,8}$.

Due to the paucibacillary nature of pleural TB, its diagnosis has emerged as a major challenge ${ }^{9}$. These diagnoses are usually made by clinical and radiological findings, although the findings are nonspecific. Conventional laboratory examinations include direct searches for Ziehl-Neelsen stain Mycobacterium tuberculosis, an assessment of cultures from biological samples such as sputum/ pleural fluid and pleural biopsies, and histopathological pleural examinations for granulomatous lesion detection ${ }^{8,10,11}$.

The conventional methods used for pleural TB diagnoses have limitations, such as low sensitivity, the time required to detect and identify mycobacteria and the need to conduct invasive procedures for biological sample collection, which can put patients at risk of bleeding, pulmonary edema and infections $\mathrm{s}^{8,10-12}$.

Additionally, pleural fluid adenosine deaminase (ADA) and interferon gamma (IFN- $\gamma$ ) levels have been proposed as biomarkers that can be used for pleural TB diagnoses, although these biomarkers may be elevated in other conditions ${ }^{5,13}$. 
Molecular tests based on M. tuberculosis genomic fragment amplification are rapid and sensitive techniques that can detect less than 10 bacilli per $\mathrm{ml}$ in a clinical sample ${ }^{14,15}$. Although conventional polymerase chain reaction (PCR) is useful for M. tuberculosis detection, the nested-PCR (nPCR) technique has greater sensitivity and specificity for the test, indicating that it is a promising laboratory tool for diagnosing paucibacillary forms of TB, including the pleural form ${ }^{6,14,16,17}$.

The aims of this study were to evaluate the performance of the nested in-house PCR technique for detection of IS6110 M. tuberculosis in clinical samples (pleural fluid, blood and urine) collected from patients with suspected pleural TB, referred by Northeast Brazil public health services, and to analyze the importance of the test in diagnosis of the disease.

\section{METHODS}

\section{Patients and samples}

A prospective, double-blinded study was conducted with 37 patients of both sexes over 15 years of age who were hospitalized for pleural effusion diagnosis investigations in public health facilities, either outsourced or from TB referral centers, in the State of Pernambuco, Northeast Brazil between August 2007 and August 2008. The association of clinical, epidemiological and laboratory criteria used by the health services-appointed physician is considered to be the gold standard when diagnosing TB. This diagnosis definition was adopted from the II Brazilian Consensus on Tuberculosis ${ }^{18}$. The laboratory criteria used to define pleural TB cases included the identification of acid-alcohol resistant bacilli (AARB) in pleural fluid or sputum samples by smear or culture or the identification of granulomatous lesions using a histopathological exam, in addition to an evident clinical anti-TB treatment response.

\section{Biological sample collection and laboratory processing}

A health services physician collected the following samples from each patient: $5.0 \mathrm{~mL}$ of pleural fluid in a sterile dry tube by thoracentesis, $4.5 \mathrm{~mL}$ of blood by venipuncture using ethylenediamine tetraacetic acid (EDTA) containing tubes (Vacutainer ${ }^{\circledR}$, Becton and Dickson, England) and $10.0 \mathrm{~mL}$ of urine in a sterile dry tube on three consecutive mornings. When possible, three successive and spontaneous 2 - to $5-\mathrm{mL}$ sputum samples were collected in the mornings, after fasting. Urine samples, but not pleural fluid or blood samples, were initially decontaminated as per the Sechi et al. protocol ${ }^{19}$.

\section{Smear}

The evaluation of sputum AARB was performed using the Ziehl-Neelsen staining technique, as per the Ministry of Health guidelines ${ }^{20}$.

\section{Mycobacterium tuberculosis culturing}

Pleural fluid samples, but not blood and urine samples, were collected in Lowenstein-Jensen medium containing tubes and were incubated at $37^{\circ} \mathrm{C}$ for in vitro growth testing.
Mycobacterium species were identified by evaluation for growth inhibition in $500 \mu \mathrm{g} / \mathrm{mL}$ para-nitrobenzoic acid (PNB) and thiophene-2-carboxylic acid hydrazide (TCH) containing media, along with niacin and $68^{\circ} \mathrm{C}$ catalase tests ${ }^{20}$.

\section{Mycobacterium tuberculosis DNA extraction and purification}

This technique was performed using a modified protocol, as first utilized by Rossetti et al. ${ }^{14,21}$. A water-containing negative control was included to assess any cross-contamination between the biological specimens.

\section{Amplification of the Mycobacterium tuberculosis IS1610 insertion sequence by nested in-house PCR}

This test was performed using a modified protocol that was first developed by Ritis et al. ${ }^{14,22}$. For each amplification, a positive control containing deoxyribonucleic acid (DNA) extracted from the M. tuberculosis H37Rv reference strain was included, along with a negative control (Milli-Q water) to exclude the possibility of cross-contamination.

\section{DNA detection method}

Ten microliters of ethidium bromide-stained PCR products was analyzed by gel electrophoresis in $2 \%$ agarose. The electrophoresis-separated DNA bands were visualized using an ultraviolet transilluminator and were photographed with a Polaroid documentation system (Kodak MP4+ System).

\section{Statistical analysis}

The collected data were analyzed using Epi-Info 6.02 (CDC, Atlanta, GA, USA). The nPCR result associations and the gold standard results were analyzed using the sensitivity, specificity and positive and negative predictive values (PPV and NPV, respectively) to assess the molecular system. Conclusions were made using a convenience sample and were based on a $5 \%$ significance level in the study population.

\section{Ethical considerations}

The research ethics were considered and approved by the Aggeu Magalhães Research Center Ethics Committee/Fundação Oswaldo Cruz (FIOCRUZ) (CAAE: 0025.0.095.000-07).

\section{RESULTS}

Of the 37 pleural effusion patients evaluated in the study, $19(51.3 \%)$ had final pleural TB diagnoses, and 18 (48.7\%) had pleural effusions due to other etiologies as established by the health services physician. The mean patient age in the study was 39 years (range: $15-78$ years); most patients were male $(57.9 \%)$, although there was no statistically significant difference between the analyzed variables.

Given the pleural TB diagnosis criteria, of the 19 pleural TB patients, 10 had a pleural fragment histopathological evaluation that was compatible with granulomatous pleuritis. In two patients who had combinations of pleural and pulmonary $\mathrm{TB}$, their sputum smears were positive in the three consecutive 
samples collected; therefore, there was no need to conduct a pleural biopsy. In four patients, the culture was positive for Koch's bacillus (KB) in the pleural fluid. Additionally, in three patients, despite inconclusive laboratory results, clinical improvements were observed following the administration of the specific therapeutic regimen recommended by the Ministry of Health ${ }^{4}$.

In 18 patients, another pathology diagnosis was made that accounted for the pleural effusion; because these patients had no TB-compatible laboratory findings, they were considered the control group. Additionally, the following notable diagnoses were also observed: uremic pleuritis, traumatic pleural effusion, neoplastic and paraneoplastic effusions, Hodgkin's lymphoma, bronchoalveolar carcinoma, chronic liver disease, rheumatoid arthritis and chronic liver disease.

A positive pleural TB diagnosis made via in-house nPCR was verified after the presence of the 316-bp amplified IS6110 insertion element was noted in the analyzed biological samples (Figure 1). The performance of the in-house nPCR in the pleural fluid, blood and/or urine samples was evaluated in 19 pleural TB patients and 18 non-pleural TB patients who were carriers of other pulmonary pathologies. The performance of the molecular technique was examined in the following situations using the gold standard clinical, epidemiological and laboratory association criteria: a result considered positive regardless of the biological sample type collected from the patient (pleural fluid, blood and/or urine) or a positive blood and/or urine specimen result and a positive pleural fluid sample result (Table 1).

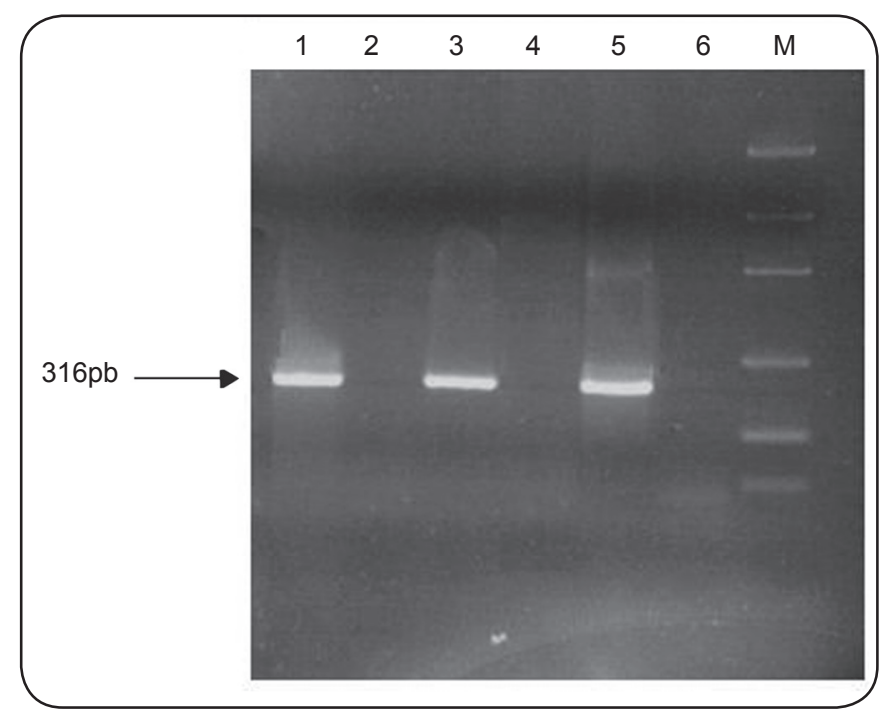

FIGURE 1 - Electrophoretic amplicon separation in a $2 \%$ agarose gel is documented across lanes 1-6. The presence of 316-bp amplicons in lanes 1 and 3 indicated the presence of the target in the blood and pleural fluid, while the amplicon was absent from the urine (Lane 2). Lanes 4 and 6 represent the negative control. Lane 5 represents the positive control (100 pg DNA - H37Rv). Lane M represents a low-molecular-weight DNA ladder.

TABLE 1 - Sensitivity, specificity and positive and negative predictive values of nested in-house PCR technique, in biological samples (blood, urine and pleural fluid) of 37 patients with suspected pleural tuberculosis.

\begin{tabular}{|c|c|c|c|c|c|c|c|}
\hline \multirow[b]{2}{*}{ Nested-PCR } & \multicolumn{7}{|c|}{ Accuracy of nested-PCR on clinical samples } \\
\hline & pleural & discarded & Total & {$[95 \% \mathrm{CI}]$} & {$[95 \% \mathrm{CI}]$} & {$[95 \% \mathrm{CI}]$} & {$[95 \% \mathrm{CI}]$} \\
\hline \multicolumn{8}{|c|}{ All the biological specimens } \\
\hline \multicolumn{8}{|c|}{ (Blood and urine and pleural fluid) } \\
\hline Total & 19 & 18 & 37 & & & & \\
\hline \multicolumn{8}{|l|}{ Pleural fluid } \\
\hline positive & 6 & 1 & 7 & $33.3 \%$ & $94.4 \%$ & $85.7 \%$ & $58.6 \%$ \\
\hline \multicolumn{8}{|c|}{ Blood and urine } \\
\hline positive & 12 & 5 & 17 & $63.2 \%$ & $72.2 \%$ & $70.6 \%$ & $65.0 \%$ \\
\hline negative & 7 & 13 & 20 & $(38.6-82.8)$ & $(46.4-89.3)$ & $(44.0-88.6)$ & $(40.9-83.7)$ \\
\hline Total & 19 & 18 & 37 & & & & \\
\hline
\end{tabular}

PCR: polymerase chain reaction; TB: tuberculosis; S: sensitivity; E: specificity; PPV: positive; NPV: negative; 95\%CI: 95\% confidence interval. 


\section{DISCUSSION}

In pleural $\mathrm{TB}$, a biopsy followed by a histopathological study and a KB culture exam is considered to be the gold standard laboratory diagnosis of this disease. However, there are significant limitations in this diagnosis method, including paucibacillary form sensitivity, the length of time required to obtain culture results, the need for patient hospitalization and the risk and discomfort inherent in the invasive biological sample collection procedures. These limitations make it difficult to obtain early diagnoses and to treat TB patients during daily clinical practice ${ }^{12,23,24}$. Therefore, it is necessary to develop a rapid and sensitive laboratory diagnosis method in which samples may be collected noninvasively and on an outpatient basis to assist in clinical management, to identify a larger number of cases and to reduce pleural TB morbidity and mortality ${ }^{25}$.

Conventional laboratory methods include direct pleural fluid examination with Ziehl-Neelsen staining of the bacillus cultures. Of the 19 patients with pleural TB in this study, direct pleural fluid microscopic analysis for KB assessment was conducted in only four patients, the results of which were negative. Direct examination is a rapid technique used in routine health services diagnoses and is considered inexpensive, but it requires a biological specimen bacillus concentration of $10,000 / \mathrm{mL}$ to obtain a positive result, with a maximum sensitivity of $5 \%$ in pleural $\mathrm{TB}^{12,20}$.

Additionally, studies indicate that culture examination is more sensitive; however, mycobacterial growth ranges from 14 days to 8 weeks ${ }^{20}$. The pleural fluid cultures were positive in only four patients (a sensitivity of $21 \%$ ). This result was likely due to the difficulty of cultivating $M$. tuberculosis, bearing in mind that this technique is able to detect 10 to 100 bacilli per $\mathrm{mL}$ of mycobacteria in these samples ${ }^{20}$. The low sensitivity in pleural fluid sample cultures was first demonstrated in 1973 by Berger \& $\mathrm{Mejia}^{26}$. This group detected mycobacteria in pleural fluid sample cultures in only $20-30 \%$ of the patients they investigated. In our study, pleural fragment culturing after biopsy was not performed. In a Brazilian study by Lima et al. ${ }^{24}$, no mycobacterial growth was observed in the fragment cultures of nine pleural TB patients who had biopsies taken.

Histopathological examinations are indicated in all exudative pleural effusion cases with unknown etiologies. It is estimated that diagnoses are made in $80 \%$ of these cases, but the procedures are not risk-free ${ }^{10-12}$. In this study, tuberculous pleuritis diagnoses were confirmed in ten patients (a sensitivity of $52.6 \%$ ) because granulomas were observed in these patients' histopathological exams. Five patients did not undergo pleural biopsies; therefore, pleural histopathological studies could not be completed. Such situations could compromise the analysis of the results because pleural biopsy is the best available method for disease diagnosis. In all cases in which a pleural biopsy was not performed, there was some clinical impediment, such as the severity of the case or a small pleural effusion volume. The performance of a pleural biopsy by needle in the presence of little or no pleural fluid can double the risk of pneumothorax or other complications during thoracentesis (from less than 5\% to approximately $11 \%)^{27}$.

In a study by Castelo Filho et al. ${ }^{18}$, it was questioned whether the use of a granulomatous finding was useful as a diagnostic criterion in tubercular exudative pleural effusion cases of unknown etiology. The authors felt that this finding may lead to a presumptive diagnosis and emphasized that these diagnoses should be based on mycobacterial isolations from the pleural fluid and sputum or in the pleural fragments. This criticism is valid in southern regions of Brazil with lower TB prevalence rates where granulomatous pleurisy is more likely to be caused by another disease (such as fungal disease, sarcoidosis or rheumatoid arthritis).

As a consequence of the various conventional laboratory method limitations for detecting M. tuberculosis, the nPCR methodology has been considered to be an alternative and auxiliary diagnostic tool that offers rapid high-specificity results while also being able to identify specific $M$. tuberculosis complex strains ${ }^{6,16,23}$.

In this study, the diagnostic performance was evaluated based on the direct detection of the IS6110 region, which is specific to the $M$. tuberculosis complex, using an in-house nPCR technique. This technique was used with pleural fluid, blood and urine clinical samples collected from pleural effusion patients suspected of having TB. In addition to pleural fluid, blood and urine specimens were also collected from each patient. These samples were also evaluated because they can be taken non-invasively, can be collected at an outpatient clinic and are alternative specimens that can be used in pleural TB diagnostic investigations.

The in-house nPCR technique sensitivity and specificity results obtained in the study exhibited variations in accordance with the positivity of the different biological samples analyzed. When the in-house nPCR technique was used to evaluate the pleural fluid samples, low sensitivity was observed (33.3\%) but was paired with high specificity (94.4\%). When the molecular technique was analyzed in blood and urine specimens simultaneously, however, an increase in sensitivity $(63.2 \%)$ and a decrease in specificity $(72.2 \%)$ were noted. As the extrapulmonary form of the disease was assessed in this study, the bacillus should circulate in all organism fluids, thus increasing the likelihood of detecting the bacillus DNA in other organism fluids/tissues.

The most satisfactory results were obtained when at least one of the pleural fluid, blood and/or urine biological samples from the same patient was considered positive based on the in-house nPCR results and had an increase in sensitivity of approximately $51 \%$ (from 33.3 to $84.2 \%$ ) when compared with the pleural fluid sample alone. In this context, despite the insufficient number of patients analyzed, we observed that a greater number of different biological sample types from the same patient that were subjected to the molecular test led to increases in sensitivity and the NPV. This finding demonstrated a tendency for a specific value that excluded the disease in our study population. 
Of the 19 patients who took part in the study and had final pleural TB diagnoses, in-house nPCR was performed on 18 pleural fluid samples, of which only six were positive. The 12 samples that returned false-negative results in the test could likely be explained by the paucibacillary nature of the samples and because the pleural fluid was an exudate arising from a hypersensitive reaction to the Mycobacterium ${ }^{16}$. Furthermore, as in other organic specimens, it is believed that pleural fluid has inhibitory substances that can interfere with the PCR reaction, thus making it necessary to adopt procedures that can maximally and simultaneously remove those inhibitors ${ }^{14,28}$. Therefore, this necessity may allow for the determination between a low copy number and the absence of the target IS6110 region in clinical isolates $^{29}$. The inhibitors that may be present in various clinical samples include hemoglobin; polysaccharides; glycoproteins; some reagents, detergents and sample preparation solvents; nitrites; urea; crystals; anticoagulants (e.g., EDTA, heparin); and certain hormones ${ }^{14,28,30}$.

Of the 18 patients who were diagnosed with another pathology, namely, non-tubercular pleurisy, a positive pleural fluid in-house nPCR result was detected for only one patient. This result was considered a false-positive nPCR result in this patient, as the gold standard evaluation did not indicate any paucibacillary forms of the disease. Additionally, this test should be evaluated together with clinical and epidemiological criteria, along with an anti-TB treatment, before the possibility of reactivation or asymptomatic disease can be determined ${ }^{31}$.

The low pleural fluid mycobacteria detection sensitivity of the nPCR technique demonstrated in this study was similar to what was observed in other studies. Lima et al. ${ }^{24}$ studied a total of 45 patients (16 with pleural TB) and reported a sensitivity of $31.3 \%$ and a specificity of $96.6 \%$. In contrast, Nagesh et al..$^{23}$ reported a sensitivity of $70 \%$ and a specificity of $100 \%$ in their study with 60 pleural effusion patients, of which 20 were due to tubercular causes. Liu et al. ${ }^{12}$ reported a sensitivity of $43.3 \%$ and a specificity of $95.5 \%$ in 163 suspected patients with negative smear tests. Kumar et al. ${ }^{6}$ obtained a sensitivity of $51.7 \%$ and a specificity of $100 \%$ in the nested nPCR results from 100 pleural effusion patients, 58 of which were tubercular in nature.

Regarding the evaluation of blood and/or urine sample inhouse nPCR performance, of the 18 non-pleural TB patients (but who were classified as carriers of other pulmonary pathologies), five positive patients were detected. These patients were considered false positives. In contrast, following clinical and epidemiological history analyses, it was determined that all of these patients had a history of previously being in contact with an adult with bacilliferous TB in the past two years; therefore, the presence of latent $\mathrm{TB}$ cannot be excluded, even if no reaction was observed with the tuberculin test, given that the patients' immunity was compromised because they were carriers of diseases that led to their hospitalization. However, in seven of the 19 patients diagnosed with pleural TB, the results were considered false negative in samples, likely because of the inhibiting substances that were contained in both of the collected (blood and/or urine) samples ${ }^{14,28}$.
In this study, an increase was observed in the positivity the blood and/or urine samples when compared with the pleural fluid samples. These findings agree with some studies, which argued for the possibility of the hematogenous spread of M. tuberculosis ${ }^{24,32}$.

Additionally, little information was available in the literature regarding urine sample assessment in PCR-based reactions for extrapulmonary TB diagnoses, except for studies evaluating PCR reactions involving the kidneys. In these studies, the in-house nPCR technique used with urine samples showed a sensitivity of $35.7 \%$ and a specificity of $100 \%$. Moreover, Kafwabulula et al..$^{33}$ observed a sensitivity of $55 \%$, while Rebollo et al. ${ }^{34}$ showed that the percentage of a positive PCR reaction in their patient urine samples was higher in human immunodeficiency virus (HIV)-positive patients than in immunocompetent patients with TB (a sensitivity of $16 \%$ was observed in this study). Cruz et al. ${ }^{14}$ obtained a sensitivity of $40 \%$ and a specificity of $90.8 \%$ in urine samples from 58 pulmonary and extrapulmonary TB patients.

In a study by Sethi et al. ${ }^{35}, M$. tuberculosis infections in extra-pulmonary specimens (22) were detected with a higher analytical sensitivity by an mpt64 reverse transcription-PCR assay when compared with the IS6110 PCR reaction (these sensitivities were $50 \%$ vs. $18 \%$, respectively). In our study, we observed a greater sensitivity in our nPCR system approach, even though the IS6110 target was used. This observation was especially true when considering that the test positivity rate from different types of samples from the same patient was $84.2 \%$ in comparison with specificity of individually observed biological specimens (which was 33\%). However, the higher IS6110 target test performance observed in our study was likely due to the nPCR system we used, which can be a useful tool for TB diagnoses in pleural and paucibacillary specimens.

Additionally, the performance of the nPCR in-house technique was increased when more than one biological sample from an individual patient was examined. When comparing the in-house nPCR M. tuberculosis detection results in pleural fluid, blood and/or urine specimens, increased sensitivity $(33.3 \%, 63.2 \%$ and $84.2 \%)$ was observed. In pleural fluid, the specificity of the technique was $94.4 \%$, which was higher than in the combined analysis of the three biological samples (72.2\%), thus confirming the limitation of traditional laboratory methods to identify patients with different clinical stages of TB (i.e., latency and disease).

Thus, the nPCR in-house technique may be used as an informative tool in pleural TB diagnoses. Additionally, considering the routine clinical associations, (including epidemiological and laboratory criteria), various sample types must be collected from the same patient so that all of them can be evaluated in the molecular test. Moreover, priority should be given to blood and urine in relation to pleural fluid. In this context, invasive procedures for diagnostic confirmation can be replaced with less-invasive sample collections, which can be taken in an outpatient clinic, thus avoiding patient hospitalizations. Finally, further studies should be undertaken using a larger group of pleural effusion patients who are not initially suspected of TB for use as negative controls. 


\section{CONFLICT OF INTEREST}

The authors declare that there is no conflict of interest.

\section{FINANCIAL SUPPORT}

This study received financial support from the Aggeu Magalhães Research Center-FIOCRUZ and the Program for Technological Development in Health Supplies (PDTIS).

\section{REFERENCES}

1. World Health Organization (WHO). Global tuberculosis control: WHO report 2011 [Internet]. Washington: World Health Organization; 2011. [cited 2013 Jan 30]. Available at: http://www.who.int/tb/publications/ global_report/2011/en/.

2. Ministério da Saúde. Sistema de Informação de Agravos de Notificação (SINAN) [Internet]. Brasília: Ministério da Saúde; 2012. [Cited 2013 Jan 30]. Available at: http://dtr2004.saude.gov.br/sinanweb/tabnet/ dh?sinannet/tuberculose/bases/tubercbrnet.def.

3. Jamal LF, Moherdau F. Tuberculose e infecção pelo HIV no Brasil: magnitude do problema e estratégias para o controle. Rev Saude Publica 2007; 41:104-110

4. Ministério da Saúde. Guia de Vigilância Epidemiológica Tuberculose [Internet]. 7th ed. Brasília: Ministério da Saúde; 2010. [Cited 2013 Jan 5]. Available at http://portal.saude.gov.br/portal/arquivos/pdf/gve_7ed_web_ atual_tuberculose.pdf.

5. Morisson P, Neves DD. Evaluation of adenosine deaminase in the diagnosis of pleural tuberculosis: a Brazilian meta-analysis. J Bras Pneumol 2008; 34:217-224.

6. Kumar P, Sen MK, Chauhan DS, Katoch VM, Singh S, Prasad HK. Assessment of the N-PCR assay in diagnosis of pleural tuberculosis: detection of M. tuberculosis in pleural fluid and sputum collected in tandem. PloS One 2010; 5:e10220.

7. Seiscento M, Conde MB, Dalcolmo MM. Tuberculous pleural effusions. J Bras Pneumol 2006; 32 (suppl IV): 174-181.

8. Gopi A, Madhaven SM, Sharma SK, Sahn SA. Diagnosis and treatment of tuberculous pleural effusion in 2006. Chest 2007; 131:880-889.

9. Pai M, Ling DI. Rapid diagnosis of extrapulmonary tuberculosis using nucleic acid amplification tests: what is the evidence? Future Microbiol 2008; 3:1-4.

10. Trajman A, Pai M, Dheda K, van Zyl Smit R, Zwerling A, Joshi R, et al. Novel tests for diagnosing tuberculous pleural effusion: What works and what does not. Eur Respir J 2008; 31:1098-1106.

11. McGrath EE, Anderson PB. Diagnostic tests for tuberculous pleural effusion. Eur J Clin Microbiol Infect Dis 2010; 29:1187-1193.

12. Liu KT, Su WJ, Perng RP. Clinical utility of polymerase chain reaction for diagnosis of smear-negative pleural tuberculosis. J Chin Med Assoc 2007; 70:148-151.

13. Yang B, Wang X, Li H, Li G, Cao Z, Cheng X. Comparison of loopmediated isothermal amplification and real-time PCR for the diagnosis of tuberculous pleurisy. Letters Appl Microbiol 2011; 53:525-531.

14. Cruz HLA, Montenegro RA, Lima JFA, Poroca DR, Lima JFC, Montenegro LML, et al. Evaluation of a nested-PCR for Mycobacterium tuberculosis detection in blood and urine samples. Braz J Microbiol 2011; 42:321-329.

15. Santos FCF, Nascimento ALA, Lira LAS, Lima JFC, Montenegro RA, Montenegro LML, et al. Bone tuberculosis: a case report on child. Rev Soc Bras Med Trop 2013; 46:249-251.

16. Asnaashari AMH, Towhidi M, Farid R, Abbaszadegan MR, Attaran D, Fatemi SS, et al. Evaluation of polymerase chain reaction for diagnosis of "Tuberculous Pleurisy". Tanaffos 2011; 10:12-18.
17. Martins LC, Paschoal IA, Von Nowakonski A, Silva SA, Costa FF, Ward LS. Nested-PCR using MPB64 fragment improves the diagnosis of pleural and meningeal tuberculosis. Rev Soc Bras Med Trop 2000; 33:253-257.

18. Castelo Filho A, Kritski AL, Barreto AW, Lemos ACM, Ruffino-Netto A, Guimarães CA, et al. II Consenso Brasileiro de Tuberculose: diretrizes brasileiras para tuberculose 2004. J Bras Pneumol 2004; 30 (suppl I): 57-86.

19. Sechi LA, Pinna MP, Sanna A, Pirina P, Ginesu F, Saba F, et al. Detection of Mycobacterium tuberculosis by PCR analysis of urine and other clinical samples from AIDS and non-HIV-infected patients. Mol Cell Probes 1997; 11:281-285.

20. Ministério da Saúde. Manual Nacional de Vigilância em Saúde e outras Micobactérias [Internet]. Brasília: Ministério da Saúde; 2008. [Cited 2009 Jun 20]. Available at: http://portal.saude.gov.br/portal/arquivos/pdf/ manual_laboratorio_tb.pdf.

21. Rossetti MLR, Jardim SB, Rodrigues VFS, Moura AR, Oliveira H, Zaha A. Improvement of Mycobacterium tuberculosis detection in clinical samples using DNA purified by glass matrix. J Microbiol Methods 1997; 28:139-146.

22. Ritis K, Tzoanopoulos D, Speletas M, Papadopoulos E, Arvanitidis K, Kartali S, et al. Amplification of IS6110 sequence of M. tuberculosis complex in HIV negative patients with Fever of Unknown Origin (FUO) and evidence of extrapulmonary disease. J Intern Med 2000; 248: 415-424.

23. Nagesh BS, Sehgal S, Jindal SK, Arora SK. Evaluation of polymerase chain reaction for the detection of Mycobacterium tuberculosis in pleural fluid. Chest 2001; 119:1737-1741

24. Lima JF, Montenegro LM, Montenegro RA, Cabral MM, Lima AS, Abath FG, et al. Performance of nested PCR in the specific detection of Mycobacterium tuberculosis complex in blood samples of pediatric patients. J Bras Pneumol 2009; 35:690-697.

25. Maurya AK, Kant S, Nag VL, Kushwaha RAS, Kumar M, Dhole TN. Comparative evaluation of IS6110 PCR via conventional methods in rapid diagnosis of new and previously treated cases of extrapulmonary tuberculosis. Tuberk Toraks 2011; 59:213-220.

26. Berger HW, Mejia E. Tuberculous pleurisy. Chest 1973; 6388-6392.

27. Colt H, Brewer N, Barbur E. Evaluation of patient-related and procedurerelated factors contributing to pneumothorax following thoracentesis. Chest 1999; 116: 134-8.

28. Santos A, Cremades R, Rodríguez JC, García-Pachón E, Ruiz M, Royo G. Comparison of methods of DNA extraction for real-time PCR in a model of pleural tuberculosis. Acta Pathol Microbiol Immunol Scand 2010; 118:60-65.

29. Mehta PK, Raj A, Singh N, Khuller GK. Diagnosis of extrapulmonary tuberculosis by PCR. FEMS Immunol Med Microbiol 2012; 66:20-36.

30. Mahony J, Chong S, Jang D, Luinstra K, Faught M, Dalby D, et al. Urine specimens from pregnant and nonpregnant women inhibitory to amplification of chlamydia trachomatis nucleic acid by PCR, ligase chain reaction, and transcription-mediated amplification: identification of urinary substances associated with inhibition and removal of inhibitory activity. J Clin Microbiol 1998; 36:3122-3126.

31. Chawla K, Gupta S, Mukhopadhyay C, Rao PS, Bhat SS. PCR for M. tuberculosis in tissue samples. J Infect Developing Countries 2009; 3:8387.

32. Backer A, Mortelé K, Keulenaer B, Parizel P. Tuberculosis: epidemiology, manifestations and the value of medical imaging in diagnosis. JBR-BTR 2006; 89:243-250

33. Kafwabulula M, Ahmed K, Nagatake T, Gotoh J, Mitarai S, Oizumi K, et al. Evaluation of PCR-based methods for the diagnosis of tuberculosis by identification of mycobacterial DNA in urine samples. Int J Tuberc Lung Dis 2002; 6:732-737.

34. Rebollo MJ, San Juan Garrido R, Folgueira D, Palenque E, Díaz-Pedroche C, Lumbreras C, et al. Blood and urine samples as useful sources for direct detection of tuberculosis by polymerase chain reaction. Diagn Microbiol Infect Dis 2006; 56:141-146.

35. Sethi S, Yadav R, Mewara A, Dhatwalia SK, Sharma M. Evaluation so in-house mpt64 real-time PCR for rapid detection of Mycobacterium tuberculosis in pulmonary and extra-pulmonary specimens. Braz J Infec Dis $2012 ; 16: 493-494$ 